\title{
Manejo del dolor irruptivo asociado a la cura de úlceras cutáneas
}

\section{Management of breakthrough pain associated with the cure of skin ulcers}

\author{
V. Domingo-Triadó1, D. López-Alarcón², F. Villegas-Estévez ${ }^{3}$, C. Alba-Moratilla ${ }^{4}$, B. Massa-Domínguez ${ }^{5}$, \\ L. Alepuz ${ }^{6}$, F. Palomar-Llatas ${ }^{2}$, A. Mínguez-Martíi, A. Sanz ${ }^{7}$, A. J. Jiménez ${ }^{7}$, L. Debón-Vicent ${ }^{8}$, \\ en representación del grupo de investigación UDODIC $\|^{\diamond}$
}

${ }^{1}$ Hospital Lluís Alcanyís, Xàtiva, Valencia, España. ${ }^{2 H o s p i t a l ~ G e n e r a l ~ U n i v e r s i t a r i o ~ d e ~ V a l e n c i a, ~ V a l e n c i a, ~}$ España. ${ }^{3}$ Consorcio Hospital Provincial de Castellón, Castellón, España. ${ }^{4}$ Hospital Clínico Universitario de Valencia, Valencia, España. ${ }^{5}$ Hospital General Universitario de Elche, España. ${ }^{6}$ Hospital de Villajoyosa,

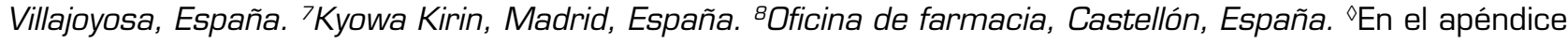
se incluyen los investigadores que pertenecen al grupo UDODIC II.

\section{ABSTRACT}

Objective: to assess the prophylactic management of breakthrough pain associated with skin ulcers care procedures, considering the different treatments used in clinical practice for the prevention and minimization of pain.

Material and methods: A multicenter, cross-sectional, observational study. The study was conducted in 11 hospitals distributed throughout Spain. Ulcer features, patient demographics and clinical characteristics were recorded. Pain assessment, and patients' anxiety and satisfaction were measured using McGill Pain Questionnaire, Hamilton Anxiety Rating Scale and visual analogue scales (VAS).

Result: Low levels of anxiety were registered [74.2\%, none or mild) and patient and healthcare professional satisfaction was high [8.3 and 7.7, respectively). Opioid drugs were administered to $73.8 \%$ of patients, who experienced significantly less pain than those who did not receive treatment or who received non-opioid drugs $(p<0.0001$ ). Type of management was related to patient age, ulcer characteristics, treatment for background pain, patient anxiety levels, perception of pain during the procedure, years of experience of the healthcare professional, the use of clinical guidelines, and

Domingo-Triadó V, López-Alarcón D, Villegas-Estévez F, Alba-Moratilla C, Massa-Dominguez B, Alepuz L, Palomar-Llatas F, Minguez-Martí A, Sanz A, Jiménez AJ, Debón-Vicent L, en representación del grupo de investigación UDODIC II. Manejo del dolor irruptivo asociado a la cura de úlceras cutáneas. Rev Soc Esp Dolor 2020;27(2):113-126

\section{RESUMEN}

Objetivos: Valorar el manejo profiláctico del dolor irruptivo asociado a la cura de úlceras cutáneas, teniendo en cuenta los diferentes tratamientos que se usan en la práctica clínica para la prevención y la reducción del dolor.

Material y métodos: Estudio multicéntrico, observacional y transversal. El estudio se llevó a cabo en 11 hospitales en España. Se recopilaron datos sobre las características de las úlceras, además de variables demográficas y clínicas de los pacientes. La valoración del dolor, la ansiedad y la satisfacción del paciente se midieron mediante el cuestionario del dolor de McGill, la escala de ansiedad de Hamilton y escalas visuales analógicas.

Resultados: La mayoría de los pacientes presentaba niveles bajos de ansiedad (74,2 \%, ninguna o leve), mientras que la satisfacción de los pacientes y los profesionales sanitarios era alta $[8,3$ y 7,7 , respectivamente). Se administraron medicamentos opioides al 73,8 \% de los pacientes, que experimentaron significativamente ( $p<0,0001$ ) menos dolor que aquellos que no recibieron tratamiento o que recibieron medicamentos no opioides. El tipo de tratamiento se relacionó con la edad del paciente, la percepción del dolor durante la cura,

Recibido: 18-07-2019

Aceptado: 18-01-2020

Correspondencia: Vicente Domingo Triadó vicente.domingo@ucv.es 
the unit in which it was performed. Factors significantly related to the use of prophylaxis were treatment for background pain, more severe ulcers, and treatment by a professional with <20 years of experience who followed clinical guidelines. Significant factors related to the use of opioids were anxiety, the care unit, and the years of experience of the healthcare professional.

Conclusión: Differences in patient management depended on numerous factors. Prophylaxis with opioids, mainly fentanyl, resulted in less pain for the patient.

Key words: Skin ulcers, preventive analgesia, opioids, wound care procedure, acute pain, pain in cures. los años de experiencia del profesional, el uso de guías clínicas y el servicio o unidad en el que se llevó a cabo la cura. El uso de profilaxis se relacionó significativamente con el tratamiento del dolor irruptivo, con úlceras más graves y con el tratamiento realizado por un profesional con menos de 20 años de experiencia, que siguió las guías clínicas. Otros factores relacionados con el uso de opioides fueron la presencia de ansiedad, la unidad donde se realizaron los cuidados y los años de experiencia del profesional de la salud.

Conclusiones: Las diferencias en el manejo del paciente dependieron de numerosos factores. La profilaxis con opioides se asoció con una menor percepción del dolor para el paciente.

Palabras clave: Úlceras cutáneas, analgesia preventiva, opioides, cura de heridas, dolor agudo, dolor en curas.

\section{INTRODUCCIÓN}

Las úlceras cutáneas son muy prevalentes en los pacientes con enfermedades crónicas. Son una de las principales causas de comorbilidad y deterioro de la calidad de vida, debido a la necesidad constante de cura y al proceso de curación, que en general es lento. Se han identificado 3 tipos principales de úlceras crónicas: úlceras por presión, vasculares (venosas y arteriales) y diabéticas. Estas son causadas por hipoxia local del tejido, infección microbiana de la úlcera, daño recurrente por isquemia-reperfusión, o por una alteración en la respuesta al estrés en las personas de edad avanzada (1). En algunos casos, las lesiones cutáneas pueden llegar a afectar áreas profundas como la hipodermis o el hueso, causando un nivel muy alto de dolor.

El dolor irruptivo, a diferencia del dolor persistente, suele ser consecuencia del daño tisular producido por los cambios regulares de vendaje y las curas o desbridamiento de las úlceras [2]. El desbridamiento consiste en retirar el tejido no viable de la úlcera y es una medida efectiva para reducir la proliferación bacteriana. La realización de desbridamientos profundos no sería posible sin un manejo adecuado del dolor. En la actualidad existen recomendaciones, pero el tratamiento del dolor durante las curas, mediante medicamentos tópicos o sistémicos, es aún muy heterogéneo en la práctica. Esto es debido, en parte, a los numerosos factores intrínsecos, extrínsecos, psicológicos y sociales involucrados (3-5). Por lo tanto, es necesario establecer protocolos de tratamiento apropiados para maximizar el alivio del dolor asociado a estos procedimientos y minimizar los efectos secundarios farmacológicos y la toxicidad $(6,7)$.

Las estrategias principales para la prevención del dolor irruptivo en pacientes con úlceras se enfocan en el uso de opioides de inicio de acción rápida y duración de la acción corta (8-12). Estos suelen administrarse 30 min antes del inicio del tratamiento local para asegurar un control adecuado del dolor. El uso de estos tratamientos, a pesar de estar fuera de indicación en algunos casos, no debería ser una opción en función del facultativo ya que tienen claras ventajas durante este tipo de procedimientos. Otras medidas para minimizar el dolor durante las curas de heridas incluyen el conocimiento del dolor existente, evitar manipulaciones innecesarias de la herida, la valoración de la piel y los tejidos adyacentes en busca de posibles infecciones o necrosis, el uso de productos para el cuidado de las úlceras a las temperaturas apropiadas y evitar la presión excesiva sobre la úlcera de los materiales de vendaje [13]. A pesar de la evidencia disponible, el impacto de los diferentes tratamientos en la prevención del dolor irruptivo y la percepción del paciente con respecto a la cura siguen sin definirse.

El objetivo principal de este estudio fue valorar el manejo profiláctico del dolor irruptivo asociado a las curas tópicas de las úlceras teniendo en cuenta los diferentes tratamientos utilizados en la práctica clínica rutinaria para la prevención o la reducción del dolor (1,14-19).

Los objetivos secundarios incluían estudiar la relación de la profilaxis con una percepción del dolor [20], evaluar la ansiedad del paciente antes de la cura, la satisfacción del paciente asociada a su experiencia con el dolor y la satisfacción del profesional sanitario por evitar el dolor al paciente.

\section{MÉTODOS}

\section{Diseño del estudio}

Estudio multicéntrico, transversal, observacional que se llevó a cabo en 11 hospitales distribuidos por toda la geografía española.

La población del estudio consistió en todos los pacientes mayores de 18 años con úlceras cutáneas y alta probabilidad de sufrir dolor durante las curas tópicas que estaban en seguimiento por parte del personal hospitalario y habían sido tratados entre mayo de 2014 y mayo de 2015. Los pacientes fueron excluidos si, en opinión del investigador, no contaban con suficiente 
capacidad cognitiva para responder a los cuestionarios. Para evitar el sesgo de selección, los pacientes que cumplian los criterios de inclusión y exclusión y que aceptaban participar aportando un consentimiento informado escrito, se incluyeron de manera secuencial.

El protocolo del estudio fue aprobado por el comité de ética del Consorcio hospitalario Provincial de Castellón el 26 de febrero de 2014. Todos los hospitales que participaron en el estudio solicitaron la aprobación por parte de las respectivas autoridades institucionales.

\section{Medición de los resultados}

Para cada paciente, se recogieron los datos del manejo profiláctico del dolor asociado a la cura tópica de las úlceras en una sola visita. Se recogió información sobre las siguientes variables mediante un cuaderno de recogida de datos:

a) Características sociodemográficas del paciente: edad, sexo, raza, nivel educativo y actividad laboral, peso, estatura e índice de masa corporal, así como antecedente médicos relevantes, las enfermedades concomitantes y su tratamiento.

b) Información de la úlcera cutánea: tiempo desde la aparición de la úlcera, etiología, localización anatómica, extensión, forma, exudado, características de la úlcera cutánea y signos de infección.

c) Historia de úlceras cutáneas (previas a la visita del estudio), tratamiento previo, número de curas tópicas.

d) Tratamiento farmacológico del dolor basal del paciente.

e) Valoración del dolor antes y durante la cura: presencia, intensidad cuantitativa del dolor y valoración cualitativa sobre el tipo y la intensidad del dolor; esto se realizó mediante el cuestionario de McGill (descrito más abajo).

f) Medidas farmacológicas y no farmacológicas utilizadas por los profesionales sanitarios para disminuir el dolor durante la cura.

g) Evaluación del dolor anticipado por los pacientes: ansiedad [Escala de Ansiedad de Hamilton [HAMA, del inglés, Hamilton Anxiety Rating Scale] descrita más abajo], satisfacción en relación con el dolor experimentado durante el tratamiento (escala visual analógica [EVA]).

h) Satisfacción de los profesionales sanitarios en términos de dolor evitado al paciente durante la cura [EVA].

El cuestionario de dolor de McGill $[21,22)$ se utilizó para valorar el dolor durante la cura, y consta de las siguientes escalas: índice de valoración del dolor (PRI, del inglés, Pain Rating Index, rango: 1-78 puntos), número de palabras elegido (NWC, del inglés, Number of Words Chosen, rango: 0-20), índice de intensidad del dolor (PPI, del inglés, Present Pain Index). El PRI es un inventario de descriptores verbales del dolor que consiste en 72 adjetivos descriptivos. El paciente revisa esta lista de descriptores de dolor y rodea aquellos que mejor describen su experiencia dolorosa actual. Cada parte o dimensión se puntúa de manera individual y la puntuación total acumulativa se suma para formar el PRI e incluye el PRI total (PRI-T, puntuación 0-78),
PRI sensorial (PRI-S, puntuación 0-42), PRI emocional (PRI-A, puntuación 0-14), PRI valorativo (PRI-E, puntuación 0-5) y PRI miscelánea (PRI-M, puntuación 0-17). El NWC es la suma del total de descriptores que elige el paciente. EI PPI se basa en una escala de intensidad del 1 al 5 (1-Leve, 2- Incómodo, 3-Estresante, 4-Horrible, 5-Insoportable).

Además, se utilizó la intensidad del dolor mediante la EVA [1: sin dolor, 10: dolor insoportable] [23), y los cambios en las puntuaciones absolutas y relativas antes y después de la cura, para evaluar la significación clínica [24]. La ansiedad durante la cura se midió usando la HAM-A [25]. Esta escala consta de 14 ítems, que miden tanto la ansiedad psicológica como la somática. Cada ítem se puntúa entre $O$ (no presente) y 4 (muy grave), con un rango total de puntuación entre 0 y 56 , en el cual la ansiedad se clasifica como: sin ansiedad (0-5), leve (6-14), moderada (15-25), grave (26-39) y muy grave (40-56). Se utilizó una escala EVA para medir la satisfacción.

\section{Análisis estadístico}

Se estimó que el tamaño de muestra necesario para identificar hasta 6 factores asociados con los diferentes perfiles de manejo del dolor durante la cura de las heridas era de 150 pacientes, con una precisión del $95 \%$ y una potencia estadística del $80 \%$.

Se realizó un análisis descriptivo de las variables recabadas. Las variables cualitativas se describieron utilizando frecuencias absolutas y porcentajes. Las variables cuantitativas que se ajustaban a una distribución normal se describieron mediante la media, la desviación estándar (DE), el mínimo y el máximo, mientras que aquellas que no se ajustaban a una distribución normal se describieron con la mediana, rango intercuartílico (primer cuartil-tercer cuartil), mínimo y máximo. Las variables categóricas se estudiaron mediante tablas de contingencia y la prueba de Chi cuadrado. Para la comparación entre variables categóricas y continuas, se estudió la distribución normal utilizando la prueba de Kolmogorov-Smirnov. Se estimó la diferencia media para las variables con distribución normal en las que la variable categórica era dicotómica, y se usó el ANOVA cuando existían más de 2 categorías. Para distribuciones no normales, se aplicaron técnicas no paramétricas (la prueba de Mann-Whitney para las dicotómicas y la prueba de Kruskal-Wallis para las variables categóricas]. Se utilizaron 2 modelos de regresión logística para determinar 1) la relación entre el tratamiento profiláctico del dolor frente al no tratamiento (variable dependiente) y las variables independientes (años de experiencia del profesional sanitario; uso de guías por parte del profesional sanitario; características de la úlcera; analgesia de base utilizada por el paciente), y 2) la asociación entre recibir tratamiento opioide frente a la ausencia de tratamiento (variable dependiente) y las variables independientes (unidad relacionada con la cura; años de experiencia: analgesia de base; HAM-A).

Se emplearon las siguientes variables para medir la intensidad del dolor: porcentaje de tiempo con dolor durante el tratamiento, es decir, la proporción de tiempo durante la cura en la cual el paciente declaró que había experimentado dolor (definido como dolor 
descontrolado, EVA $\geq 4$ ); intensidad relativa del dolor durante el tratamiento comparada con la del dolor de base, es decir, el resultado obtenido de la división de la intensidad del dolor durante la cura entre la intensidad del dolor de base; e intensidad relativa del dolor, obtenida de multiplicar las 2 variables previas. Se realizó un análisis por subgrupos, combinando las siguientes variables, que mostraron significación estadística con el test ANOVA: profundidad de la úlcera [con 4 estadios: superficial, menos $1 \mathrm{~cm}$, entre 1 y $3 \mathrm{~cm}$, más de $3 \mathrm{~cm}$ ), exudado (con 4 estadios: sin exudado, leve, moderado, grave] y signos de infección [ 2 estadios: sí/ no), para identificar 2 grupos bien diferenciados: los pacientes con úlceras "más graves" y "menos graves".
Se realizó un contraste de hipótesis bilateral con un nivel de significación del 0,05. Se utilizaron los programas SAS y SPSS v22 9.4 para el análisis estadístico de los datos.

\section{RESULTADOS}

\section{Características principales de los pacientes, las úlceras y las curas}

Como muestra la Tabla I, los 124 pacientes del estudio tenían una edad media de 70,5 $\pm 13,2$ años y una distribución equilibrada entre géneros (el $52 \%$ eran

TABLA I

DESCRIPCIÓN DE CARACTERÍSTICAS PRINCIPALES

\begin{tabular}{|c|c|}
\hline Características de los pacientes & $N[\%]$ \\
\hline Edad (media $\pm \mathrm{DE})$ & $70,5 \pm 13,2$ \\
\hline Sexo (hombres), n [\%] & $64(51,6)$ \\
\hline Peso $(\mathrm{kg})$ (media \pm DE) & $77,2 \pm 17,7$ \\
\hline IMC (media \pm DE) & $28,1 \pm 6,1$ \\
\hline \multicolumn{2}{|l|}{ Historia clínica } \\
\hline Hipertensión, n [\%] & $63[50,8)$ \\
\hline Anomalías de la coagulación, $n$ [\%] & $15(12,1)$ \\
\hline Enfermedades concomitantes, $n[\%]$ & $118(95,2)$ \\
\hline Diabetes, $n[\%]$ & $47(37,9)$ \\
\hline Insuficiencia vascular, n [\%] & $76(61,3)$ \\
\hline Aterosclerosis, n [\%] & $36(29,0)$ \\
\hline Tumores, n [\%] & $10(8,1)$ \\
\hline \multicolumn{2}{|l|}{ Ansiedad en relación con la cura actual } \\
\hline Escala HAM-A, media (DE) & $10,0(8,0)$ \\
\hline Ansiedad psicológica, media (DE) & $6,8[5,4)$ \\
\hline Ansiedad somática, media (DE) & $3,2[3,6]$ \\
\hline \multicolumn{2}{|l|}{ Unidades/Servicios hospitalarios } \\
\hline Cirugía vascular, n [\%] & $25(20,7)$ \\
\hline Cirugía general, n [\%] & $13(10,7)$ \\
\hline Cuidados domiciliarios, n [\%] & $23(19,0)$ \\
\hline Dolor, n [\%] & $29(24,0)$ \\
\hline Úlceras, n [\%] & $41(33,9)$ \\
\hline Atención Primaria, n [\%] & $10[8,3]$ \\
\hline Otros, n [\%] & $49(40,5)$ \\
\hline \multicolumn{2}{|l|}{ Profesionales } \\
\hline Enfermeras, n (\%) & $107(92,2)$ \\
\hline Médicos, n [\%] & $9(7,8)$ \\
\hline Uso de guías clínicas & $74(60,7)$ \\
\hline \multicolumn{2}{|l|}{ Años de experiencia } \\
\hline$\leq 10, \mathrm{n}[\%]$ & $21(14,4)$ \\
\hline 11-20, n [\%] & $37[30,6]$ \\
\hline$>20, n[\%]$ & $63(52,1)$ \\
\hline
\end{tabular}

DE: desviación estándar. HAM-A: Escala de Ansiedad de Hamilton. IMC: índice de masa corporal. 
hombres]. En general, los pacientes tenían sobrepeso (índice de masa corporal 28,1 $\pm 13,0 \mathrm{~kg} / \mathrm{m}^{2}$ ), el $51 \%$ eran hipertensos y la mayoría sufría de enfermedades concomitantes $[95,0 \%)$, siendo las más prevalentes la insuficiencia vascular $(61,0 \%)$ y la diabetes $[38,0 \%)$.

Los profesionales sanitarios que realizaron las curas fueron en su mayoría enfermeras (92,2 \%); el 60,7\% siguió las guías clínicas ${ }^{3-5}$ y el 82,6 \% contaba con más de 10 años de experiencia. Además de las unidades de atención primaria (8,3\%), las curas se realizaron principalmente en las unidades de úlceras [33,9\%), las unidades de dolor $[24,0 \%$ y los servicios de cirugía vascular $[20,7 \%]$, o en las unidades de atención domiciliaria (19,0\%). Surgieron complicaciones en el 5,6 \% de los pacientes: hubo 3 casos de amputación, uno de inflamación, uno de dolor/ansiedad, un caso de inflamación e infección concomitante y otro paciente presentó sangrado leve durante el desbridamiento. Las características relevantes de las úlceras se muestran en la Figura 1.
Tratamiento del dolor persistente y del dolor irruptivo procedimental durante las curas previas

En total, el 79,8 \% de los pacientes recibía tratamiento analgésico para el manejo del dolor persistente. Casi la mitad tomaba medicamentos no opioides $[49,5 \%$ ), el 19,2 \% tomaba opioides, y el 31,3\% recibía ambos tipos de medicamentos (Figura 2). Además, el 87,1\% de los pacientes había sido sometido a una cura de úlceras previamente, y la mitad a más de 29 curas de úlceras (mediana de 29,5; rango intercuartílico de 6-67,5). Un total del 60,2 \% de los pacientes que habían sido sometidos a una cura de úlceras había recibido tratamiento analgésico durante la cura: el 67,2 \% con medicamentos no opioides, el 25,0\% opioides, y el 7,8\% restante había recibido ambos tipos de medicación (Figura 2). Entre los medicamentos no opioides, los más utilizados fueron el paracetamol $[56,3 \%$ y el metamizol $[46,3 \%]$ para el dolor persistente, y el metamizol [56,3 \%) y

Porcentaje de úlceras

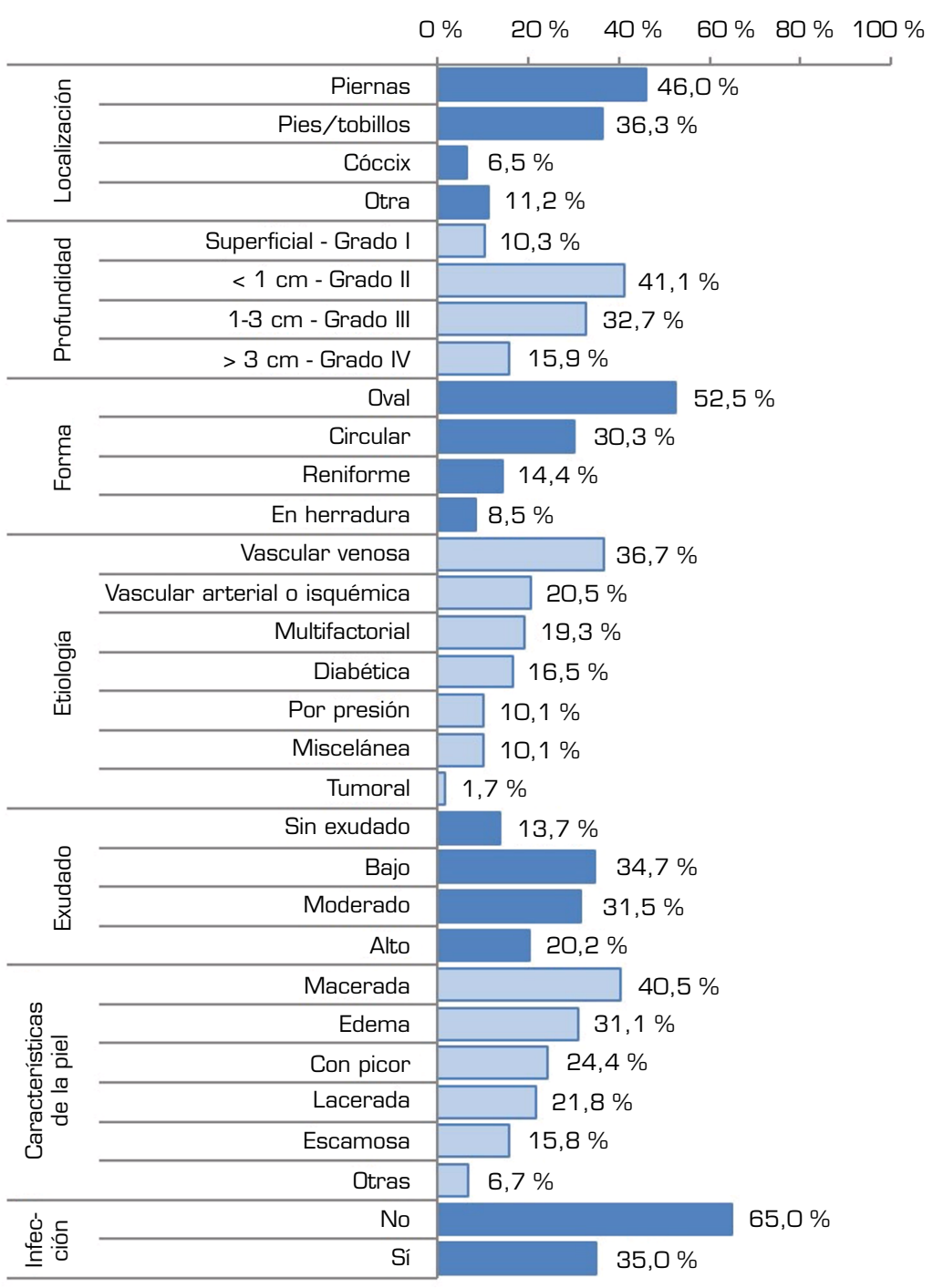

Fig. 1. Características de las úlceras. 
el paracetamol $(37,5 \%$ ) en curas de úlceras previas. De los opioides administrados, el fentanilo (32,0\%), el tramadol $[28,0 \%$ y y la combinación tramadol/paracetamol [26,0\%) se utilizaron con mayor frecuencia para el dolor persistente, mientras que el tramadol [33,3\%], la morfina [28,6\%), el fentanilo (19,0\%) y la combinación tramadol/paracetamol (14,3\%) se habían utilizado en curas previas de úlceras (Figura 3).

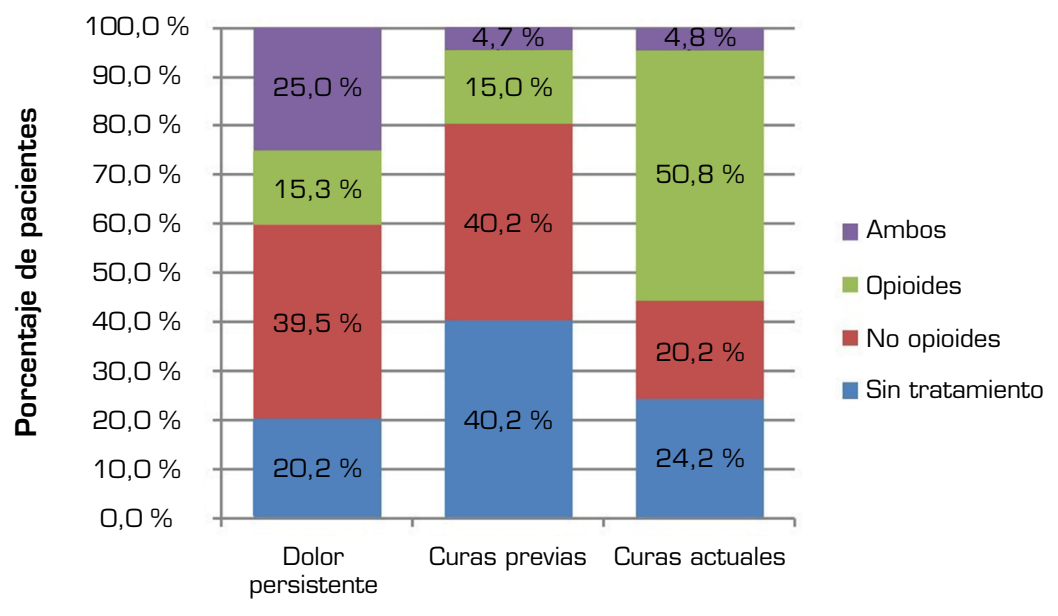

Fig. 2. Comparación entre el tratamiento para el manejo del dolor persistente y el del dolor irruptivo en las curas previas y actuales (faltan datos para 16 de las curas previas y para 6 de las actuales).

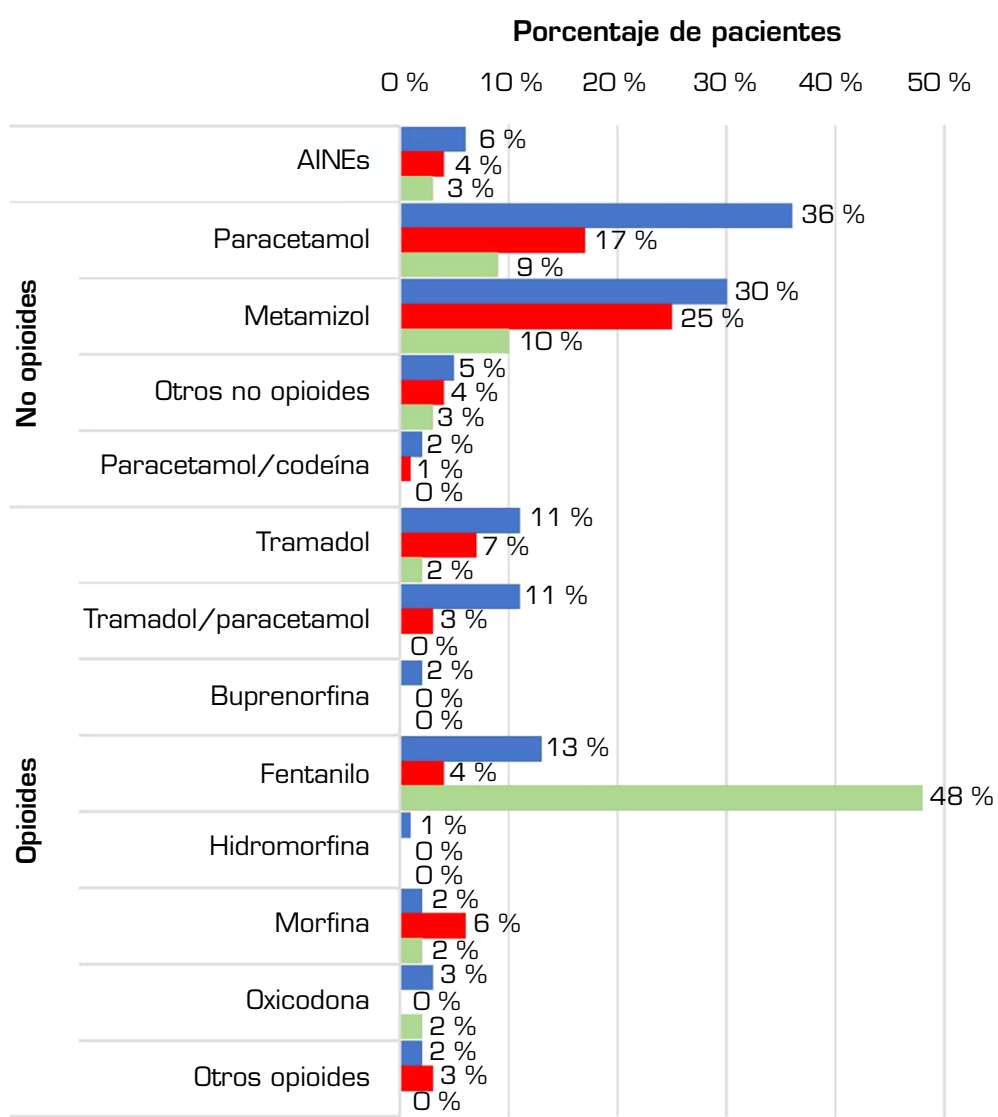

Medicación utilizada para el dolor persistente

- Medicación utilizada en curas previas

Medicación utilizada en la cura actual

Fig. 3. Diferentes tipos de medicación utilizados para el dolor persistente y el dolor irruptivo en curas previas y actuales. Los porcentajes se basan en el número total de pacientes para los cuales había información disponible: 108 para curas previas, 124 para dolor persistente y 124 para curas actuales. 


\section{Tratamiento durante las curas del estudio}

Un tercio de los pacientes fueron tratados en su domicilio [33,0\%]. La duración de la cura fue de 27,8 min de media (DE: 20,7). Se administró tratamiento analgésico en 94 pacientes (75,8 \%); 69 recibieron medicamentos opioides (63 pacientes como agente único y 6 en combinación con otros tratamientos), mientras que 25 pacientes recibieron medicamentos no opioides (Figura 2). El fentanilo fue el opioide más utilizado durante las curas $[93,8 \%$ de aquellos tratados únicamente con opioide, $n=59$ ) (Figura 3). De los 59 pacientes tratados con fentanilo, 42 (71,2 \%) recibieron la formulación sublingual, 9 [15,3\%] la formulación oral, 2 (3,4 \%) utilizaron los parches transdérmicos y un paciente $[1,7 \%)$ recibió fentanilo intranasal. Todos los pacientes que recibieron fentanilo fueron previamente evaluados por un médico especialista que aprobó el uso de este producto. Además, las enfermeras que lo administraron estaban entrenadas en el manejo de las posibles complicaciones. Comparando las curas del estudio con las curas previas, los opioides, y el fentanilo en particular, se emplearon con una frecuencia signifi- cativamente mayor en las curas del estudio $(p<0,001$ para ambas comparaciones].

\section{Factores asociados al manejo profiláctico}

Para comparar el manejo del dolor irruptivo asociado con las curas del estudio, los tipos de profilaxis se clasificaron en 4 categorías: sin tratamiento (30 pacientes; 24,2 \%), tratamiento no opioide (17 pacientes; $13,7 \%$ ), tratamiento solo con opioides o combinado con otros productos (63 pacientes; $50,8 \%$ ) y anestesia (14 pacientes; 11,3\%). Como se ha mencionado anteriormente, las características de la úlcera (profundidad, exudado, infección] se combinaron en una única variable.

El análisis univariante reveló que el tipo de manejo profiláctico de la úlcera se asociaba significativamente con la edad, las características de la úlcera, el tipo de analgesia utilizada para el dolor persistente, el nivel de ansiedad de acuerdo con la HAM-A, la variación del dolor experimentado durante la cura, los años de experiencia del profesional sanitario, la unidad en la que se llevó a cabo la cura y el uso de guías clínicas durante la cura [Tabla II].

TABLA II

ANÁLISIS UNIVARIANTE DE LAS VARIABLES SIGNIFICATIVAS ASOCIADAS AL TRATAMIENTO PROFILÁCTICO DEL DOLOR

\begin{tabular}{|c|c|c|c|c|c|}
\hline & Global & $\stackrel{\operatorname{Sin}}{\text { tratamiento }}$ & No opioides & Opioides & Valor de $\mathrm{p}$ \\
\hline \multicolumn{5}{|l|}{ Edad } & \multirow{4}{*}{0,042} \\
\hline $\mathrm{N}$ & 122 & 30 & 17 & 61 & \\
\hline$\leq 69$ & $41,0 \%$ & $46,7 \%$ & $41,2 \%$ & $31,1 \%$ & \\
\hline$>69$ & $59,0 \%$ & 53,3 \% & 58,8 \% & 68,9 \% & \\
\hline \multicolumn{5}{|c|}{ Características de la úlcera } & \multirow{4}{*}{0,001} \\
\hline $\mathrm{N}$ & 124 & 30 & 17 & 63 & \\
\hline Menos grave & $54,8 \%$ & $70,0 \%$ & $58,8 \%$ & $57,1 \%$ & \\
\hline Más grave & $45,2 \%$ & $30,0 \%$ & $41,2 \%$ & $42,9 \%$ & \\
\hline \multicolumn{5}{|c|}{ Analgesia de base utilizada por el paciente } & \multirow{5}{*}{$<0,001$} \\
\hline $\mathrm{N}$ & 124 & 30 & 17 & 63 & \\
\hline Sin tratamiento & $20,2 \%$ & $43,3 \%$ & $5,90 \%$ & $14,3 \%$ & \\
\hline No opioides & $39,5 \%$ & $40,0 \%$ & $76,5 \%$ & $27,0 \%$ & \\
\hline Opioides & $40,3 \%$ & $16,7 \%$ & $17,6 \%$ & $58,7 \%$ & \\
\hline \multicolumn{5}{|c|}{ Escala de ansiedad de Hamilton } & \multirow{7}{*}{$<0,001$} \\
\hline $\mathrm{N}$ & 120 & 28 & 17 & 61 & \\
\hline Sin ansiedad & $36,7 \%$ & $60,7 \%$ & $52,9 \%$ & $13,1 \%$ & \\
\hline Ansiedad leve & $37,5 \%$ & $35,7 \%$ & $29,4 \%$ & $42,6 \%$ & \\
\hline Ansiedad moderada & $20,0 \%$ & $3,6 \%$ & $5,9 \%$ & $36,1 \%$ & \\
\hline Ansiedad grave & $5,8 \%$ & $0,0 \%$ & $11,8 \%$ & $8,2 \%$ & \\
\hline Ansiedad muy grave & $0,0 \%$ & $0,0 \%$ & $0,0 \%$ & $0,0 \%$ & \\
\hline
\end{tabular}


TABLA II [CONT.]

ANÁLISIS UNIVARIANTE DE LAS VARIABLES SIGNIFICATIVAS ASOCIADAS AL TRATAMIENTO PROFILÁCTICO DEL DOLOR

\begin{tabular}{|c|c|c|c|c|c|}
\hline & Global & $\begin{array}{c}\text { Sin } \\
\text { tratamiento }\end{array}$ & No opioides & Opioides & Valor de $\mathrm{p}$ \\
\hline \multicolumn{5}{|c|}{ Cambios en el dolor con el manejo profiláctico } & \multirow{5}{*}{$<0,001$} \\
\hline $\mathrm{N}$ & 123 & 30 & 17 & 62 & \\
\hline Menos dolor & $47,2 \%$ & $20,0 \%$ & $47,1 \%$ & $64,5 \%$ & \\
\hline Mismo dolor & $41,5 \%$ & $56,7 \%$ & $47,1 \%$ & $33,9 \%$ & \\
\hline Más dolor & $11,4 \%$ & $23,3 \%$ & $5,9 \%$ & $1,6 \%$ & \\
\hline \multicolumn{5}{|c|}{ Años de experiencia del profesional sanitario } & \multirow{5}{*}{$<0,001$} \\
\hline $\mathrm{N}$ & 121 & 29 & 16 & 62 & \\
\hline$\leq 10$ & $17,4 \%$ & $6,9 \%$ & $0,0 \%$ & $30,6 \%$ & \\
\hline $11-20$ & $30,6 \%$ & $10,3 \%$ & $18,8 \%$ & $45,2 \%$ & \\
\hline$>20$ & $52,1 \%$ & $82,8 \%$ & $81,3 \%$ & $24,2 \%$ & \\
\hline \multicolumn{5}{|c|}{ Tipo de profesional sanitario involucrado en la cura } & \multirow{6}{*}{0,002} \\
\hline $\mathrm{N}$ & 121 & 29 & 17 & 61 & \\
\hline Atención primara & $16,5 \%$ & $13,8 \%$ & $11,8 \%$ & $23,0 \%$ & \\
\hline Atención domiciliaria & $14,9 \%$ & $20,7 \%$ & $0,0 \%$ & $14,8 \%$ & \\
\hline Úlcera & $48,8 \%$ & $27,6 \%$ & $47,1 \%$ & $55,7 \%$ & \\
\hline Cirugía & $19,8 \%$ & $37,9 \%$ & $41,2 \%$ & $6,6 \%$ & \\
\hline \multicolumn{5}{|c|}{ Uso de guías por parte del profesional sanitario } & \multirow{4}{*}{0,016} \\
\hline $\mathrm{N}$ & 122 & 28 & 17 & 63 & \\
\hline No & $39,3 \%$ & $46,4 \%$ & $23,5 \%$ & $47,6 \%$ & \\
\hline Sí & $60,7 \%$ & $53,6 \%$ & $76,5 \%$ & $52,4 \%$ & \\
\hline
\end{tabular}

Las variables asociadas significativamente a la administración del tratamiento para el dolor fueron el uso de guías clínicas, los años de experiencia del profesional sanitario, el tratamiento del dolor persistente y las úlceras clasificadas como más graves. Los factores asociados con el uso de opioides durante la cura fueron la unidad en la que se realizó la cura, los años de experiencia del profesional sanitario y la presencia de ansiedad. El hecho de recibir tratamiento para el dolor persistente mostró una tendencia a la asociación con el uso de opioides durante la cura [Tabla III].

\section{Valoración del dolor}

Antes de la cura, el 76,6\% de los pacientes refería dolor incontrolable (EVA $\geq 4$ ). La puntuación media de intensidad de dolor fue de 6,0 (DE: 3,3). La puntuación del PRI era de 29,9 (DE: 22,1) y el NWC era de 12,3 (DE: 8,3). De acuerdo con el PPI, las palabras que reflejaban mejor la magnitud del dolor durante las curas previas fueron estresante [26,6 \%] u horrible [33,0\%] (Figura 4).

En cuanto al dolor experimentado durante la cura del estudio, más de la mitad de los pacientes refirieron ausencia de dolor o dolor controlado (EVA < 4,5\%); el 47,2 \% declaró una reducción en el dolor, mientras que el $41,5 \%$ de los pacientes no experimentaron ninguna variación en la intensidad de su dolor. La media de intensidad del dolor (de acuerdo con la EVA) fue de 3,8 (DE: 2,8). El PRI fue de 19,5 (DE: 19,1) y el NWC fue de 10,4 (DE: 8,6). Según el PPI, las palabras que mejor reflejaron la magnitud del dolor en ese momento fueron leve (26\%) o incómodo (48\%). Entre los pacientes que sufrieron dolor durante la cura, el tiempo medio en el que experimentaron dolor fue del $50 \%$ (DE: $31 \%$ ), con una intensidad relativa del dolor durante el proceso de cura de 0,8 (DE: 0,85 según la EVA) y una puntuación media de dolor relativo de 0,3 (DE: 0,33).

Si se compara la cura del estudio con las curas previas en términos de dolor experimentado, los pacientes mostraron un dolor significativamente menor durante la cura del estudio frente al dolor sufrido en las curas previas, para todos los instrumentos: PRI, PPI, NWC, EVA. La HAM-A para la evaluación de ansiedad, alcanzó una puntuación media de 10 entre los pacientes que fueron sometidos a las curas (DE: 8,02; mín 0, máx 32): una media de 6,8 (DE: 5,4 ) para los síntomas psicológicos y una media de 3,2 [DE: 3,6] para los somáticos. La puntuación total de esta escala, una vez categorizada, reflejó un nivel de ansiedad de 0 [36, $7 \%$ ] o leve $[37,5 \%]$. 
TABLA III

REGRESIÓN LOGÍSTICA CON LOS FACTORES RELACIONADOS SIGNIFICATIVAMENTE CON EL TRATAMIENTO PROFILÁCTICO O CON EL TRATAMIENTO CON OPIOIDES

\begin{tabular}{|c|c|c|c|c|c|c|}
\hline & Coeficiente & Error estándar & Wald & Valor de $p$ & OR & IC $95 \%$ \\
\hline \multicolumn{7}{|c|}{ Tratamiento profiláctico vs. ausencia de tratamiento } \\
\hline \multicolumn{7}{|c|}{ Años de experiencia del profesional sanitario } \\
\hline 0 a 10 & & & & ref & & \\
\hline $11-20$ & 0,186 & 1,298 & 0,021 & 0,886 & 1,2 & $0,1-15,3$ \\
\hline$>20$ & $-3,032$ & 1,288 & 5,542 & 0,019 & 0,048 & $0,004-0,6$ \\
\hline \multicolumn{7}{|c|}{ Uso de guías por parte del profesional sanitario } \\
\hline No & & & & ref & & \\
\hline Sí & 3,638 & 1,168 & 9,701 & 0,002 & 38 & 3,9-375,3 \\
\hline \multicolumn{7}{|c|}{ Características de la úlcera } \\
\hline Menos grave & & & & ref & & \\
\hline Más grave & 1,568 & 0,744 & 4,444 & 0,035 & 4,8 & $1,1-20,6$ \\
\hline \multicolumn{7}{|c|}{ Analgesia de base utilizada por el paciente } \\
\hline Sin tratamiento & & & & ref & & \\
\hline No opioides & 2,383 & 0,882 & 7,303 & 0,007 & 10,8 & $1,9-61,1$ \\
\hline Opioides/Ambos & 3,44 & 1,12 & 9,426 & 0,002 & 31,2 & $3,5-280,1$ \\
\hline \multicolumn{7}{|c|}{ Tratamiento con opioides vs. cualquier otro tipo de manejo } \\
\hline \multicolumn{7}{|c|}{ Unidad involucrada en la cura } \\
\hline Atención primaria & & & & ref & & \\
\hline Atención domiciliaria & 1,437 & 1,006 & 2,04 & 0,153 & 0,238 & 0,59-30,3 \\
\hline Úlcera & 2,016 & 0,998 & 4,084 & 0,043 & 0,133 & $1,1-53,1$ \\
\hline Cirugía & 3,971 & 1,189 & 11,147 & 0,001 & 0,019 & $5,2-545,6$ \\
\hline \multicolumn{7}{|l|}{ Años de experiencia } \\
\hline $0-10$ & & & & ref & & \\
\hline $11-20$ & 1,275 & 1,048 & 1,48 & 0,224 & 0,279 & $0,46-27,9$ \\
\hline$>20$ & 3,044 & 1,049 & 8,426 & 0,004 & 0,048 & $2,7-164$ \\
\hline \multicolumn{7}{|l|}{ Analgesia de base } \\
\hline Sin tratamiento & & & & ref & & \\
\hline No opioides & 0,520 & 0,862 & 0,364 & 0,546 & 0,595 & $0,3-9,1$ \\
\hline Opioides/Ambos & $-1,687$ & 0,961 & 3,085 & 0,079 & 5,4 & $0,03-1,2$ \\
\hline \multicolumn{7}{|c|}{ Escala de ansiedad de Hamilton } \\
\hline Sin ansiedad & & & & ref & & \\
\hline Ansiedad leve & $-1,408$ & 0,719 & 3,838 & 0,05 & 4,09 & $0,06-1,001$ \\
\hline Ansiedad moderada & $-3,663$ & 1,153 & 10,094 & 0,001 & 38,98 & $0,003-0,246$ \\
\hline Ansiedad grave & $-2,922$ & 1,385 & 4,451 & 0,035 & 18,59 & 0,004-0,813 \\
\hline
\end{tabular}

IC $95 \%$ : intervalo de confianza del $95 \%$. OR: odds ratio. 

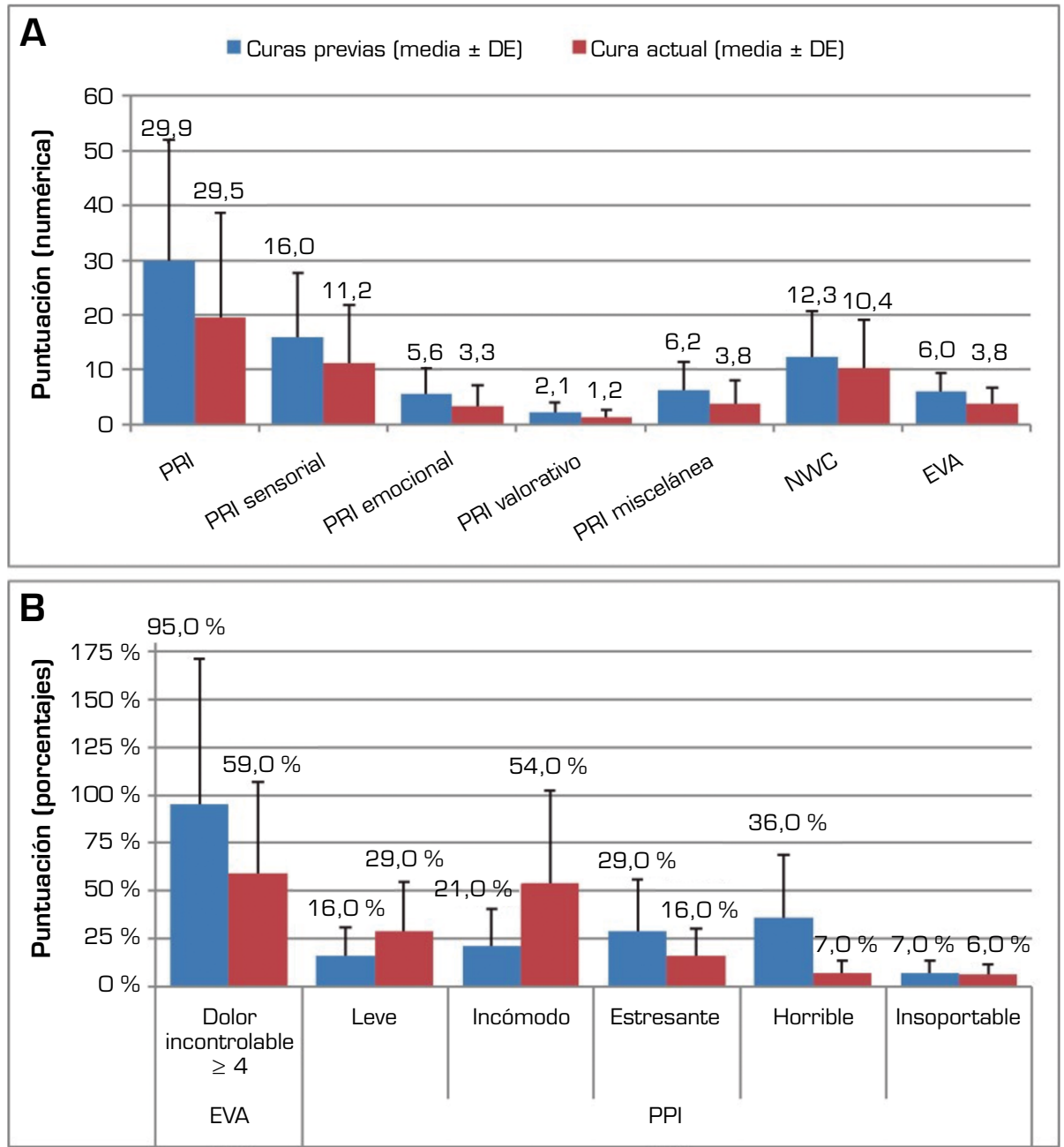

Fig. 4. Características del dolor de acuerdo con los diferentes cuestionarios. Comparación entre las curas previas y las actuales.

A. Puntuación numérica. Todas las comparativas fueron estadísticamente significativas $(p<0,001$, prueba de los rangos con signo de Wilcoxon).

B. Puntuación en porcentaje. Todas las comparativas fueron estadísticamente significativas: dolor incontrolable $\geq 4$ EVA, $p=0,0017$ (test de McNemar); PPI, $p<0,001$ (prueba de tendencias de Cochran-Armitage).

DE: desviación estándar; EVA: escala visual analógica; NWC: número de palabras elegido; PPI: índice de intensidad del dolor; PRI: índice de valoración del dolor.

Aquellos que recibieron tratamiento profiláctico con opioides experimentaron significativamente menos dolor que otros grupos (una reducción del dolor del $64,5 \%$ comparado con el $20-47 \%$ de otros tipos de manejo, $p<0,0001$ ), mientras que la proporción de pacientes con alivio y sin percepción de cambio fue similar entre aquellos que no fueron tratados con opioides (Tabla IV).

\section{Satisfacción del paciente y del profesional}

La puntuación media de satisfacción del paciente (rango: 0 a 10) fue de 8,3. Los pacientes estaban más satisfechos con el manejo por parte del profesional sanitario (el 61,8\% estaba completamente satisfecho) y el tiempo de duración del proceso $(59,7 \%$ ) que con la intensidad del dolor durante el tratamiento $[47,1 \%$ ] y la eficacia del tratamiento médico $[42,3 \%$ ).

Los profesionales sanitarios declararon estar más satisfechos con el control del dolor (45,5\%) y con el manejo de los pacientes durante el tratamiento (50\%) que con el tiempo empleado en el mismo [38,8\%]. La puntuación media de satisfacción declarada por los profesionales sanitarios en relación con la cura fue de 7,7 puntos (en una escala de $\mathrm{O}$ a 10).

Se observó una asociación positiva moderada entre las 2 variables (coeficiente de Spearman 0,521; 
TABLA IV

FACTORES ASOCIADOS SIGNIFICATIVAMENTE CON EL DOLOR DE ACUERDO CON EL MANEJO PROFILÁCTICO

\begin{tabular}{|c|c|c|c|c|}
\hline & $\begin{array}{c}\text { Sin tratamiento } \\
n=30\end{array}$ & $\begin{array}{c}\text { No opioides } \\
n=17\end{array}$ & $\begin{array}{c}\text { Opioides } \\
n=63\end{array}$ & Valor de $p$ \\
\hline \multicolumn{4}{|c|}{ Pacientes con variación del dolor n [\%] } & \multirow{5}{*}{$<0,0001$} \\
\hline $\mathrm{n}$ & 30 & 17 & 62 & \\
\hline Menos dolor & $6(20,0)$ & $8(47,1)$ & $40(64,5)$ & \\
\hline Mismo dolor & $17(56,7)$ & $8(47,1)$ & $21(33,9)$ & \\
\hline Más dolor & $7(23,3)$ & $1(5,9)$ & $1(1,6)$ & \\
\hline \multicolumn{4}{|c|}{ Intensidad relativa del dolor durante la cura } & \multirow{5}{*}{0,009} \\
\hline $\mathrm{n}$ & 19 & 17 & 59 & \\
\hline Media $\pm \mathrm{DE}$ & $1,1 \pm 1,2$ & $0,7 \pm 0,5$ & $0,5 \pm 0,3$ & \\
\hline$[Q 1 ; Q 3]$ & {$[0,2 ; 2]$} & {$[0,43 ; 0,9]$} & {$[0,3 ; 0,7]$} & \\
\hline Mín; Máx & $0 ; 4$ & $0 ; 2,25$ & $0 ; 1,6$ & \\
\hline \multicolumn{4}{|c|}{ Cambio en la puntuación del PRI } & \multirow{5}{*}{$<0,0001$} \\
\hline $\mathrm{n}$ & 30 & 17 & 63 & \\
\hline Media $\pm \mathrm{DE}$ & $-2,9 \pm 11,9$ & $-7,4 \pm 9,1$ & $-17,9 \pm 18,3$ & \\
\hline [Q1-Q3] & {$[-9,2]$} & {$[-15,0]$} & {$[-34,0]$} & \\
\hline Mín, Máx & $-25,25$ & $-26,2$ & $-54,12$ & \\
\hline \multicolumn{4}{|c|}{ Cambio en la intensidad de acuerdo con el PPI* } & \multirow{5}{*}{$<0,0001$} \\
\hline $\mathrm{n}$ & 18 & 17 & 60 & \\
\hline Menos intensidad & $5(0,3 \%)$ & $4[0,2 \%]$ & 49 (0,8\%) & \\
\hline Misma intensidad & $9(0,5 \%)$ & $12[0,7 \%]$ & $8(0,1 \%)$ & \\
\hline Más intensidad & $4[0,2 \%]$ & $1[0,1 \%]$ & $3(0,1 \%)$ & \\
\hline \multicolumn{4}{|c|}{ Cambios en la puntuación EVA } & \multirow{5}{*}{$<0,0001$} \\
\hline $\mathrm{n}$ & 30 & 17 & 62 & \\
\hline Media $\pm \mathrm{DE}$ & $-0,37 \pm 3,46$ & $-1,76 \pm 2,50$ & $-3,82 \pm 2,60$ & \\
\hline$[Q 1, Q 3]$ & {$[-2,2]$} & {$[-3,-1]$} & {$[-6,-2]$} & \\
\hline Mín, Máx & $-10,6$ & $-6,5$ & $-9,3$ & \\
\hline \multicolumn{4}{|c|}{ Cambio relativo en la puntuación PRI } & \multirow{5}{*}{0,001} \\
\hline $\mathrm{n}$ & 20 & 17 & 48 & \\
\hline Media $\pm \mathrm{DE}$ & $-20,7 \pm 57,5$ & $-22,0 \pm 31,8$ & $-54,7 \pm 36,4$ & \\
\hline [Q1; Q3] & {$[-67,3 ; 17,3]$} & {$[-46,8 ; 0,0]$} & {$[-80,6 ;-28,3]$} & \\
\hline Mín, Máx & $-100,100$ & $-80,50$ & $-100,57,1$ & \\
\hline \multicolumn{4}{|c|}{ Cambio relativo en la puntuación EVA } & \multirow{5}{*}{0,009} \\
\hline $\mathrm{n}$ & 19 & 17 & 59 & \\
\hline Media \pm DE & $9,92 \pm 118,58$ & $-26,27 \pm 50,57$ & $-50,59 \pm 29,30$ & \\
\hline [Q1-Q3] & {$[-80,100]$} & {$[-57,1,-11,1]$} & {$[-66,7,-33,3]$} & \\
\hline Min-Max & $-100,300$ & $-100,125$ & $-100,60$ & \\
\hline
\end{tabular}

DE: desviación estándar. EVA: escala visual analógica. PPI: índice de intensidad del dolor. PRI: índice de valoración del dolor. Q1: cuartil 1. Q3: cuartil 3.

Los valores de $\mathrm{p}$ se han obtenido comparando los grupos presentados en columnas mediante Chi cuadrado en el caso de frecuencias (porcentajes) y ANOVA en el caso de variables continuas (medias y desviaciones estándar). 
$p<0,0001$ ]. Por lo tanto, a mayor satisfacción del paciente, mayor satisfacción del profesional sanitario.

\section{DISCUSIÓN}

La evidencia demuestra que la prevalencia, la gravedad o las características del dolor asociado con la cura de úlceras varían mucho $[7,26,27]$. En nuestro estudio, la percepción general de los pacientes fue que el dolor durante la cura se había mantenido o reducido con respecto a experiencias anteriores, incluso en aquellos casos en los que los pacientes sintieron que las curas eran dolorosas e informaron de la existencia de dolor durante la cura. Por otro lado, se administraron opioides con mayor frecuencia en las curas de este estudio que en las realizadas anteriormente o para el tratamiento del dolor persistente, sobre todo en el caso de la administración de fentanilo. Además, cuando se administraban opioides, los pacientes sentían significativamente menos dolor.

Antes de la cura del estudio, se calificaba más frecuentemente al dolor como estresante u horrible, mientras que después se describía como leve o irritante. Es más, los pacientes presentaban niveles leves o moderados de ansiedad durante la cura del estudio. De esta manera, el grado de satisfacción tanto de los pacientes como de los profesionales en relación con las curas fue alto.

Un estudio reciente ha demostrado que el manejo inadecuado del dolor es un factor asociado con una baja calidad de vida relacionada con la salud (28). En este sentido, el nivel general de satisfacción observado en nuestro estudio confirma que el manejo del dolor irruptivo fue, en general, óptimo.

La disponibilidad de los fentanilos ha tenido un alto impacto en las estrategias de tratamiento profiláctico utilizadas por los profesionales sanitarios. Este hecho se ha constatado en este estudio, ya que el uso de fentanilo ha sido más frecuente en la visita del estudio en comparación con las curas previas. La seguridad y la eficacia del fentanilo en el manejo del dolor durante la cura de heridas se evaluó en un estudio abierto, multicéntrico, prospectivo, no controlado (12) donde se observó que la terapia con este opioide proporcionaba un alivio del dolor clínicamente significativo (para el dolor por movimiento y por curas] con menos efectos secundarios. Adicionalmente, un estudio observacional transversal [29] realizado en unidades del dolor y de úlceras mostró que los pacientes $(n=20)$ tratados con fentanilo sintieron significativamente menos dolor irruptivo durante la cura tópica de sus heridas, en comparación con otros analgésicos tales como el paracetamol y/o el metamizol.

El presente estudio revela que los pacientes que no recibieron analgesia fueron en su mayoría aquellos que se encontraban en mejores condiciones, con úlceras menos graves, que, o bien no estaban recibiendo analgesia de base, o bien solo recibían analgesia no opiácea, y que no presentaban ansiedad. Estos pacientes se distribuían de manera uniforme entre las diferentes unidades y generalmente eran tratados por profesionales con más de 20 años de experiencia.

Por el contrario, los pacientes manejados con anestesia presentaban úlceras más graves y recibían analgesia de base (bien con opioides, bien con medicamentos no opioides]. La asociación entre el dolor y el estadio de la úlcera ha sido demostrada en otros estudios [26,30]. Estos pacientes tenían en su mayor parte menos de 69 años, fueron tratados en unidades de úlceras, también por profesionales con más de 20 años de experiencia y que utilizaban guías clínicas.

El uso de una estrategia de analgesia profiláctica durante la cura (sea con opioides o no) fue más común entre los profesionales que seguían las guías clínicas y aquellos con menos de 20 años de experiencia profesional. Esta estrategia estaba orientada a pacientes con úlceras más graves y que estaban recibiendo tratamiento farmacológico para manejar el dolor persistente. Específicamente, los opioides profilácticos se administraron principalmente a pacientes con un nivel moderado de ansiedad, con mayor frecuencia en unidades de úlceras y servicios quirúrgicos y por parte de profesionales con menos de 20 años de experiencia. Por todo ello, podemos decir que los resultados del objetivo primario de este estudio definen de forma clara los aspectos referentes al manejo profiláctico del dolor irruptivo asociado a las curas, así como los resultados de los objetivos secundarios aportan una visión detallada de la percepción de paciente y profesional en función de dicho manejo. Las variables que condicionan estos aspectos deberían tenerse en cuenta a la hora de establecer estrategias y protocolos de rutina en la práctica clínica, lo cual puede repercutir de forma positiva en el abordaje futuro del dolor asociado a estos procedimientos.

Nuestro estudio tiene limitaciones evidentes. Por un lado, se trata de un estudio observacional, de modo que los pacientes no fueron aleatorizados sino reclutados de manera secuencial. Asimismo, es un estudio multicéntrico y los pacientes del estudio se trataron en diferentes localizaciones clínicas, lo que resulta en una cohorte de pacientes heterogénea. Tanto los diagnósticos de úlceras como los servicios hospitalarios involucrados en su tratamiento eran muy variados. Además, la naturaleza transversal del estudio nos impide sacar conclusiones firmes. Por lo tanto, son necesarios más estudios prospectivos aleatorizados para explorar el manejo del dolor en estos pacientes, comparando diferentes modalidades de tratamiento y sus potenciales complicaciones.

\section{CONCLUSIONES}

En resumen, en línea de otros estudios $[31,32]$, el manejo del dolor irruptivo durante las curas de úlceras depende de varios factores intrínsecos y extrínsecos, ambos relacionados con el paciente y con el profesional sanitario. En general, las unidades más especializadas manejan los casos más graves, y el uso de opioides resultó en menos dolor para el paciente.

\section{APÉNDICE}

Los siguientes investigadores pertenecen al grupo UDODIC II: Ángel Duato Jané, Hospital Clínico Lozano Blesa, Zaragoza, España; Dolores Hinojosa Caballero, 
Elena Espejo, Xenia Garrigos, Lorenzo Álvarez, M. ${ }^{\text {a }}$ Antonia Pol Reyes, Hospital Consorci Sanitari Terrassa, Barcelona, España; Eulalia Villegas Bruguera, Centre Mèdic Trauma, Barcelona, España; Diego de Alcalá Martínez Gómez, Hospital Morales Meseguer, Murcia, España; María Ángeles Segarra, Carolina Peralta, María Florentina Pitarch, Hospital de Vinaroz, Castellón, España; María Pesudo Gil, Hospital la Magdalena, Castellón, España; Emilio Garijo Gómez, Hospital Doctor Peset, Valencia, España.

\section{FUENTES DE FINANCIACIÓN}

Estudio impulsado por los investigadores y financiado por Kyowa Kirin Farmacéutica S. L. U.

\section{AGRADECIMIENTOS}

Se trata de un estudio impulsado por los investigadores y financiado por Kyowa Kirin Farmacéutica S. L.U. Todos los autores colaboraron en la recopilación de información y en su interpretación y tuvieron acceso a toda la información y a los informes.

\section{BIBLIOGRAFÍA}

1. Mustoe TA, O'Shaughnessy K, Kloeters O. Chronic wound pathogenesis and current treatment strategies: $A$ unifying hypothesis. Plastic Reconstr Surg. 2006;117(7 Suppl):35S41S. DOI: 10.1097/01.prs.0000225431.63010.1b.

2. Payne R. Recognition and diagnosis of breakthrough pain. Pain Med. 2007;8(Suppl 1):S3-S7. DOI: 10.1111/j.15264637.2006.00269.x.

3. National Pressure Ulcer Advisory Panel (NPUAP), European Pressure Ulcer Advisory Panel (EPUAP), Pan Pacific Pressure Injury Alliance (PPPIA), editores. Prevention and treatment of pressure ulcers: clinical practice guideline. Perth, Australia: Cambridge Media; 2014. Disponible en: https://www.npuap.org/wp-content/uploads/2014/08/ Updated-10-16-14-Quick-Reference-Guide-DIGITAL-NPUAPEPUAP-PPPIA-160ct2014.pdf

4. National Institute for Health and Care Excellence UK (NICE) The prevention and management of pressure ulcers in primary and secondary care. NICE clinical guidelines. London: NICE; 2014.

5. Qaseem A, Mir TP, Starkey M, Denberg TD. Risk assessment and prevention of pressure ulcers: a clinical practice guideline from the American College of Physicians. Ann Intern Med. 2015;162(5):359-69. DOI: 10.7326/M14-1567.

6. Bennett D, Burton AW, Fishman S, Fortner B, McCarberg $\mathrm{B}$, Miaskowski C, et al. Consensus panel recommendations for the assessment and management of breakthrough pain: Part 2 management. P and T. 2005;30(6):354-61.

7. Magnani C, Giannarelli D, Casale G. Procedural pain in palliative care: is it breakthrough pain? A multicenter national prospective study to assess prevalence, intensity, and treatment of procedure-related pain in patients with advanced disease. Clin J Pain. 2017;33(8):707-14. DOI: 10.1097/AJP.0000000000000450.

8. Cánovas Martínez ML, Rodríguez Rodríguez AB, Castro Bande M, Pérez Arviza L, López Soto C, Román Nuñez
R. Treatment of breakthrough pain. Rev Soc Esp Dolor. 2012;19(6):318-24.

9. Giuggioli D, Manfredi A, Vacchi C, Sebastiani M, Spinella A, Ferri C. Procedural pain management in the treatment of scleroderma digital ulcers. Clin Exp Rheumatol. 2015;33(1):5-10.

10. Bastami S, Frodin T, AhIner J, Uppugunduri S. Topical morphine gel in the treatment of painful leg ulcers, a double-blind, placebo-controlled clinical trial: a pilot study. Int Wound J. 2012;9(4):419-27. DOI: 10.1111/j.1742481X.2011.00901.x.

11. Argoff CE. Topical analgesics in the management of acute and chronic pain. Mayo Clin Proc. 2013;88(2):195-205. DOI: 10.1016/j.mayocp.2012.11.015.

12. Domingo-Triado V, Lopez Alarcon MD, Villegas Estevez F, Alba Moratillas C, Massa Dominguez B, Palomares Paya F, et al. [Breakthrough pain treatment with sublingual fentanyl in patients with chronic cutaneous ulcers]. Rev Esp Anestesiol Reanim. 2014;61(8):429-33.

13. World Union of Wound Healing Societies. Principles of best practice: minimising pain at wound dressing-related procedures. A consensus document. 2007.

14. Pellicano R, Guerra L. [Skin ulcer pain]. Minerva Med. 2012;103(6):525-31.

15. Rook JL. Wound care pain management. ADv Wound Care. 1996;9(6):24-31.

16. Rosenthal D, Murphy F, Gottschalk R, Baxter M, Lycka B, Nevin K. Using a topical anaesthetic cream to reduce pain during sharp debridement of chronic leg ulcers. J Wound Care. 2001;10(1):503-05. DOI: 10.12968/ jowc.2001.10.1.26042

17. Vernassiere C, Cornet C, Trechot P, Alla F, Truchetet F, Cuny $\mathrm{JF}$, et al. Study to determine the efficacy of topical morphine on painful chronic skin ulcers. J Wound Care. 2005;14(6):28993. DOI: 10.12968/jowc.2005.14.6.26793.

18. Panuncialman J, Falanga V. Unusual causes of cutaneous ulceration. Surg Clin North Am. 2010;90(6):1161-80. DOI: 10.1016/j.suc.2010.08.006.

19. Scimeca CL, Fisher TK, Bharara M, Armstrong DG. Chronic, painful lower extremity wounds: postoperative pain management through the use of continuous infusion of regional anaesthesia supplied by a portable pump device. Int Wound J. 2010;7(3):195-8. DOI: 10.1111/j.1742481X.2010.00675.x.

20. Cepeda MS, Africano JM, Polo R, Alcala R, Carr DB. What decline in pain intensity is meaningful to patients with acute pain? Pain. 2003;105(1-2):151-57.

21. Melzack R. The McGill Pain Questionnaire: major properties and scoring methods. Pain. 1975;1(3):277-99. DOI: 10.1016/0304-3959(75)90044-5.

22. Walters SJ, Morrell CJ, Dixon S. Measuring health-related quality of life in patients with venous leg ulcers. Qual Life Res. 1999;8(4):327-36. DOI: 10.1023/A:1008992006845.

23. Price DD, Barrell JJ, Gracely RH. A psychophysical analysis of experimential factors that selectively influence the affective dimension of pain. Pain. 1980;8(2):137-49. DOI: 10.1016/0304-3959(88)90001-2.

24. Farrar JT, Young Jr JP, LaMoreaux L, Werth JL, Poole RM. Clinical importance of changes in chronic pain intensity measured on an 11-point numerical pain rating scale. Pain. 2001;94(2):149-58. DOI: 10.1016/S03043959(01)00349-9.

25. Lobo A, Chamorro L, Luque A, Dal-Re R, Badia X, Baro E, et al. [Validation of the Spanish versions of the Montgomery- 
Asberg depression and Hamilton anxiety rating scales]. Med Clin (Barc). 2002;118(13):493-99. DOI: 10.1016/SO0257753(02)72429-9.

26. Girouard K, Harrison MB, VanDenKerkof E. The symptom of pain with pressure ulcers: A review of the literature. Ost Wound Manag. 2008;54(5):30-42.

27. Gorecki C, Closs SJ, Nixon J, Briggs M. Patient-reported pressure ulcer pain: A mixed-methods systematic review. J Pain Sympt Manag. 2011;42(3):443-59. DOI: 10.1016/j. jpainsymman.2010.11.016.

28. Herberger K, Rustenbach SJ, Haartje 0, Blome C, Franzke N, Schafer I, et al. Quality of life and satisfaction of patients with leg ulcers-results of a community-based study. Vasa. 2011;40(2):131-38. DOI: 10.1024/0301-1526/ $\mathrm{aOO0083.}$
29. Palomar Llatas F, Fornes Pujalte B, Sierra Talamantes C, Millá Tamarit I, Debon Vicent L. A cross-sectional observational study of the management of breakthrough pain associated with the treatments in patients with cutaneous ulcers. Enferm Dermatol. 2016;10(27):36-42.

30. Hopman WM, Buchanan M, VanDenKerkhof EG, Harrison MB. Pain and health-related quality of life in people with chronic leg ulcers. Chronic Dis Inj Can. 2013;33(3):167-74.

31. Schneider I, Keller A, Fleischer S, Berg A. "And there are always enough 'promo leaflets' ". A qualitative study on the situation of leg ulcer patients and their needs in regard to patient education. Pflege Z. 2013;66(8):488-93.

32. Herber OR, Schnepp W, Rieger MA. A systematic review on the impact of leg ulceration on patients' quality of life. Health Qual Life Outcomes. 2007;5:44. DOI: 10.1186/1477-7525-5-44. 